Title:

\title{
Characterization of Advanced Electronic Materials
}

Author(s): Aloysius J. Arko, Robert H. Heffner, Michael F. Hundley, Albert Migliori, Joe D. Thompson, Thomas Graf, Lianping Le, David Mandrus and Roman Movshovich, MST-10; Zachary Fisk, Florida State University; Jon M. Lawrence, University of California, Irvine; Sjoerd S. Mentink, University of Toronto; and Norman E. Phillips, University of Califomia, Berkeley

Submitted to: DOE Office of Scientific and Technical Information (OSTI)

\section{DISCLAIMER}

\begin{abstract}
This report was prepared as an account of work sponsored by an agency of the United States Government. Neither the United States Government nor any agency thereof, nor any of their empleyees, makes any warranty, express or implied, or assumes any legal liability or responsibility for the accuracy, completeness, or usefulness of any information, apparatus, product, or process disclosed, or represents that its use would not infringe privately owned rights. Reference herein to any specific commercial product, process, or service by trade name, trademark, manufacturer, or otherwise does not necessarily constitute or imply its endorsement, recommendation, or favoring by the United States Government or any agency thereof. The views and opinions of authors expressed herein do not necessarily state or reflect those of the United States Government or any agency thereof.
\end{abstract}

\section{DSTPIBUTION OF THS DOCUMENT IS UNLMTED}

\section{Los Alamos}
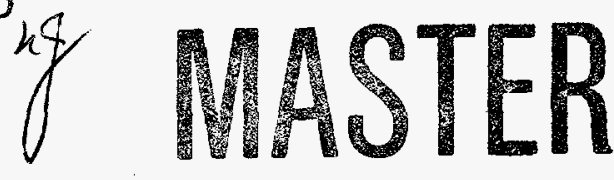

NATIONAL LABORATORY

Los Alamos National Laboratory, an affimative action/equal opportunity employer, is operated by the University of Calitomia for the U.S. Department of Energy under contract W-7405-ENG-36. By acceptance of this article, the publisher recognizes that the U.S. Government retains a nonexchustve, royaltytree license to publish or reproduce the published form of this contribution, or lo allow others to do so, for U.S. Government purposes. Los Alamos National Laboratory requests that the publisher idently this article as work periormed under the auspices of the U.S. Department of Energy. Los Alamos Natlonal Laboratory strongly supports academic freedom and a researcher's right to publish; as an institution, however, the Laboratory does not endorse the viewpoint of a publication or guarantee its technical correctness. 


\section{DISCLAMIER}

Portions of this document may be illegible in electronic image products. Images are produced from the best available original documert. 


\title{
Characterization of Advanced Electronic Materials
}

\author{
Aloysius J. Arko, Robert H. Heffner, Michael F. Hundley, Albert Migliori, Joe D. \\ Thompson*, Thomas Graf, Lianping Le, David Mandrus and Roman Movshovich \\ Materials Science Technology Division, Los Alamos National Laboratory \\ Zachary Fisk \\ Department of Physics, Florida State University \\ Jon M. Lawrence \\ Department of Physics,University of California, Irvine \\ Sjoerd S. Mentink \\ Department of Physics, University of Toronto \\ Norman E. Phillips \\ Department of Chemistry, University of California, Berkeley
}

\begin{abstract}
This is the final report of a three-year, Laboratory-Directed Research and Development (LDRD) project at the Los Alamos National Laboratory (LANL). Our goal has been to extend the Laboratory's competency in nuclear and advanced materials by characterizing (measuring and interpreting) physical properties of advanced electronic materials and in this process to bridge the gap between materials synthesis and theoretical understanding. Attention has focused on discovering new physics by understanding the ground states of materials in which electronic correlations dominate their properties. Among several accomplishments, we have discovered and interpreted pressure-induced superconductivity in $\mathrm{CeRh} \mathrm{Si}_{2}$, the competition between magnetism and superconductivity as a function of boron content in $\mathrm{UBe}_{13-\mathrm{x}} \mathrm{B}_{\mathrm{x}}$ and the origin of small gaps in the spin and charge excitation spectra of $\mathrm{Ce}_{3} \mathrm{Bi}_{4} \mathrm{Pt}_{3}$, and we provided seminal understanding of large magnetoresistive effects in $\mathrm{La}_{1-x} \mathrm{Ca}_{x} \mathrm{MnO}_{3}$. This work has established new research directions at LANL and elsewhere, involved numerous collaborators from throughout the world and attracted several postdoctoral fellows.
\end{abstract}

\section{Background and Research Objectives}

Los Alamos has had a very strong competency in materials, particularly in processing, forming and prototyping devices based on nuclear or other hazardous materials. This competency, developed over the last several decades, was critical for ensuring national security. The nation will continue to need this competency in the future, but there is growing realization that a basic understanding of materials at the electronic level is required to ensure the safety and reliability of our nuclear stockpile. Additionally,

\footnotetext{
${ }^{*}$ Principal Investigator, E-mail: jdt@mst.lanl.gov
} 
attention is shifting more toward advanced electronic materials that will strengthen the country's competitive posture and that will allow development of technologies to limit proliferation of weapons and environmental pollution. These are substantially new directions for the Laboratory that require a change in emphasis from what has been primarily on metallurgy to electronic properties of materials, i.e. modern experimental and theoretical physics of condensed matter. In this role, we must develop capabilities to probe electronic interactions in detail and couple results of experimental research to theoretical models.

Electronic properties of materials are determined not only by the electronic configuration but also by the complex interplay among electronic, magnetic and lattice (structural) interactions. The extent of this complexity is exemplified in materials ranging from plutonium to high temperature superconductors. Only through systematic, in-depth characterization of high quality materials is there hope of revealing the fundamentals of these materials. This is the central point: materials characterization is at the heart of a Laboratory competency in advanced materials; it provides the key link between materials synthesis and theoretical understanding. To build and extend the Laboratory's competency in characterization of nuclear and advanced materials, we must develop a comprehensive suite of capabilities that will probe electronic, magnetic and lattice interactions at microscopic and macroscopic levels. One of our objectives has been the development of this capability. The second and primary objective has been the application of this capability to measure and interpret physical properties of advanced electronic materials important to ongoing Laboratory programs. Materials in which strong electronic correlations have a dominant role in determining physical properties are common to several Laboratory programs pursued by researchers in T, LANSCE, CST, NMT and MST Divisions as well as centers within these divisions. Quite generally, strongly correlated electron systems typify the complex interplay among electronic, magnetic and structural interactions, and as a whole they represent a substantial fraction of the Laboratory's entire effort in advanced materials. As a means of developing competency in nuclear and advanced materials, we have focused our second objective on understanding the ground states of correlated electron materials.

\section{Importance to LANL's Science and Technology Base and National R\&D Needs}

Our effort to develop characterization expertise and capabilities on the forefront of research is applicable to the study of wide varieties of advanced electronic materials. Our principal aim is to strengthen the Laboratory's competency in nuclear and advanced 
materials by providing a basis for understanding the complex interplay among electronic, magnetic and structural interactions responsible for the unique properties of these materials and to thereby position Los Alamos as the lead DOE laboratory in this important area. In part, we had already played significant roles in developing new, advanced materials initiatives at Los Alamos, including those in high temperature superconductivity, the National High Magnetic Field Laboratory (NHMFL) at Los Alamos and the Correlated Electron Theory Program. Without our core expertise in materials characterization, the probability of success of these new initiatives would have been reduced substantially.

During the course of this three-year project, we used our characterization expertise to make seminal studies of the lanthanum manganites that were discovered to exhibit a very large magnetoresistance. These early, key experiments led to Los Alamos' teaming with Hewlett-Packard to host the nation's first workshop on the physics and applications of these correlated electron materials, a workshop that was very valuable in helping set a national agenda in this area and, more generally, in the study of spin-charge-lattice coupling in complex electronic materials. A direct outgrowth of the latter has been the revitalization of plutonium science at Los Alamos, with renewed emphasis on fundamental understanding at the electronic level. A further spinoff from this LDRD project has come from our work on characterizing the ground state of materials in which there is a small semiconducting energy gap whose origin is in electronic correlations. In the course of this research, we realized that these and related materials might be useful in creating a next generation of thermoelectric/magneto-thermoelectric cooling devices. This effort is now a well-defined project supported by DOE/AEP at Los Alamos and has created a similar project at Oak Ridge National Laboratory. Though both of these new projects are just beginning, they hold substantial promise for enabling entirely new industrial and defense technologies that require reliable, efficient solid-state cooling.

\section{Scientific Approach and Accomplishments}

The need to develop competency in measuring and interpreting physical properties of advanced materials should be driven by scientific as well as technical objectives. Because strongly correlated electron materials are a prominent theme in the Laboratory's overall advanced materials effort, we have focused particularly on characterizing and understanding this important class of materials that is at the forefront of condensed matter science worldwide. Heavy-fermion compounds are prototypes of strong electronic correlations and were used as the means to exercise our characterization capabilities while addressing important scientific problems. However, as better or more technologically 
relevant examples of advanced materials were discovered during the course of the program, we responded appropriately to these developments.

There are two particularly unusual ground states realized in heavy-fermion materials which, if understood, would mark major advances in the area of correlated electron phenomena. Interestingly, these ground states are diametrically opposed, one being superconducting and the other semiconducting. In most heavy-fermion superconductors, superconductivity coexists with some form of weak magnetism that results in complex pressure-, temperature-, and field-dependent phases. This has led to the speculation that magnetic interactions may mediate superconducting electron-pair formation. If true, these materials exhibit entirely new superconducting ground states that are not describable by conventional theory in which phonons produce pair formation. Understanding this ground state is a significant experimental and theoretical challenge and may bear directly on high- $T_{c}$ superconductivity, which is believed by many to have its origin in magnetic interactions as well. At the other extreme is a semiconducting gap found in some heavy-fermion materials that is anomalously small $(\sim 100 \mathrm{~K})$ relative to more conventional semiconductors like silicon or germanium. In spite of this, some researchers have argued that the small gap in heavy-fermion systems is simply a consequence of electronic band structure. However, our work establishes unambiguously that this is not the case but that the small gap is a direct consequence of electronic correlations; in fact, correlations that may not be qualitatively different from those that produce a heavy-fermion superconductivity. The small semiconducting gap seems to appear when the ratio of localized electrons to itinerant electrons is exactly unity. This is a special case of the generalized heavy-fermion problem that is more straightforwardly handled theoretically.

Careful, in-depth characterization of high quality samples is a particularly powerful approach to revealing the essential interactions and their interplay. To this end, we have applied, as appropriate, expertise in characterization that includes: macroscopic characterization through specific heat from 0.05 to $20 \mathrm{~K}$, magnetic susceptibility from 1.8 to $350 \mathrm{~K}$ in fields to $5 \mathrm{~T}$, electrical resistance from $0.05 \mathrm{~K}$ to $300 \mathrm{~K}$ at pressures to 100 kbar and fields to $10 \mathrm{~T}$, thermoelectric power and thermal expansion from 1.5 to $300 \mathrm{~K}$, resonant ultrasound spectroscopy above $2 \mathrm{~K}$, muon spin relaxation ( $\mu \mathrm{SR})$ at dilution refrigerator temperatures, nuclear magnetic resonance/nuclear quadrupole resonance (NMR/NQR), photoemission spectroscopy above $20 \mathrm{~K}$, neutron diffraction and $\mathrm{x}$-ray absorption spectroscopy above $15 \mathrm{~K}$. We have developed pressure capabilities for specific heat in collaboration with the University of California, Berkeley, as well as high field capabilities for specific heat, electrical resistance and thermal expansion. High magnetic fields are particularly important for studying the small-gap semiconductors because, aside 
from temperature, this is the only thermodynamical variable that has an energy scale comparable to the gap value $\left(1 \mathrm{~T} / \mu_{\mathrm{B}} \approx 1 \mathrm{~K} / \mathrm{k}_{\mathrm{B}}\right)$.

During the course of this three-year project we studied many different correlated electron systems, and this work is reported in publications listed with this report. The following highlights significant accomplishments in three areas.

(a) Correlated electron superconductivity: $\mathrm{UBe}_{13}$ is a superconductor below $\mathrm{T}_{\mathrm{C}}=$ $0.9 \mathrm{~K}$ and is strongly correlated as indicated by its thermodynamic effective electron mass, which is nearly 1000 times larger than the mass of a free electron. As expected for a superconductor in which the electron-electron pairing mechanism is unconventional, properties of this material are very sensitive to nonmagnetic as well as magnetic impurities. Careful studies as a function of impurity content allow us to investigate the pairing mechanism and symmetry of the superconducting ground state order parameter. We have found that very small substitutions of $B$ for $B e$ in $U_{B e_{13-x}} B_{x}(x=0.01,0.03,0.044$, $0.068,0.09,0.11)$ drastically alter both normal and superconducting properties. Analysis of specific heat $C_{p}$ data near and below $T_{C}$ revealed a factor of two increase in the $C_{p}$ anomaly at $T_{C}$ with initial additions of $B$, signifying a huge increase in coupling between electrons and boson excitations (most likely, spin fluctuations) mediating the superconductivity. ${ }^{11} \mathrm{~B}$ and ${ }^{9} \mathrm{~B}$ NMR Knight shift data are temperature independent below $\mathrm{T}_{\mathrm{C}}$, contrary to a Knight shift that decreases exponentially in conventional superconductors having s-wave order-parameter symmetry. This is strong evidence for higher-order (e.g. $d$-wave) symmetry. Further, from $\mu$ SR measurements on $x=0.09$ and $x=0.11$ samples, we have discovered very weak magnetic order $\left(\mu<0.001 \mu_{B}\right)$ that develops below $T_{C}$ and coexists with superconductivity. Finally, an interpretation of resistivity and specific heat data above $T_{C}$ is consistent with the characteristic spin-fluctuation temperature dropping below $T_{c}$ for $x \geq 0.05$. Together these measurements argued for $U B e_{13-x} B_{x}$ being a $d$-wave superconductor in which superconductivity is mediated by spin fluctuations that become static on the time scale of $\mu$ SR experiments $(\leq 1 \mu \mathrm{s})$ as the fluctuation-temperature scale drops below $\mathrm{T}_{\mathrm{C}}$.

Closely related is our work on $\mathrm{CeRh}_{2} \mathrm{Si}_{2}$ which is an antiferromagnet with a Néel temperature $T_{N}=35 \mathrm{~K}$ at atmospheric pressure. From our earlier study, we knew that $T_{N}$ could be driven to $T=0$ at a critical pressure $P_{C}=9 \mathrm{kbar}$, and at this pressure the crystallographic unit-cell volume was very close to that of $\mathrm{CeCu}_{2} \mathrm{Si}_{2}$, which is a heavyfermion superconductor at ambient pressure. This led to our speculation that $\mathrm{CeRh}_{2} \mathrm{Si}_{2}$ at 9 kbar also might superconduct. Indeed, we discovered that superconductivity appears (below $400 \mathrm{mK}$ ) very near the critical pressure required to suppress long-range antiferromagnetic order. Further, specific heat measurements under pressure are fully 
consistent with significant local spin fluctuations at $P \geq P_{c}$. Not only is this perhaps the strongest evidence yet for spin-fluctuation-mediated superconductivity in heavy-fermion materials, but is also emphasizes the interplay among electronic, magnetic, and lattice (i.e., volume) interactions in these materials.

(b) Small gap semiconductors: Electrical resistance and thermopower measurements on $\mathrm{Ce}_{3} \mathrm{Bi}_{4} \mathrm{Pt}_{3}$ reveal a gap in its charge-excitation spectrum $\mathrm{E}_{\mathrm{g}} \approx 100 \mathrm{~K}$. ${ }^{209} \mathrm{Bi}$ NMR/NQR experiments showed that the NQR resonance frequency $v_{Q}$ departed from metallic-like to insulating-like behavior near $T_{M}=80 \mathrm{~K}$ where the static susceptibility is a maximum. The NMR Knight shift had both isotropic and arrisotropic components, anisotropy that arises from the transferred hyperfine coupling between Ce-4f and conduction electrons A change in scaling between the static susceptibility and both the isotropic and axial Knight shifts at $T_{M}$ provided strong evidence that hybridization between $4 \mathrm{f}$ orbitals and conduction electrons is responsible for the gap structure. This view was supported by thermal expansion, magnetostriction and high-temperature-specific heat data. A Gruneissen analysis of these data, which considers the lattice coupling to spin and charge degrees-of-freedom, revealed, however, that a magnetic field-induced volume change was not equivalent to a temperature-induced volume change. This is unusual and suggested that charge and spin excitations are inequivalent in this materials, even though both are strongly coupled to the lattice. This conclusion was supported from a comparison of the effect of $\mathrm{La}$ substitution in $\left(\mathrm{Ce}_{1-x} \mathrm{La}_{\mathrm{x}}\right)_{3} \mathrm{Bi}_{4} \mathrm{Pt}_{3}$ on the specific heat and the neutron quasi-elastic scattering response. Together these studies provided unambiguous evidence that the small semiconducting gap in $\mathrm{Ce}_{3} \mathrm{Bi}_{4} \mathrm{Pt}_{3}$ is not conventional, i.e., is not a simple band structure effect, but develops from the strong interplay among magnetic, electronic and lattice interactions in this correlated electron system.

(c) Magnetoresistive materials: Research on correlated electron superconductors and semiconductors was temporarily interrupted to respond to a report in late 1994 of the observation of a very large negative magnetoresistance in doped lanthanum manganite. This report and its potential technological implication for sensor applications were so compelling that our characterization effort was redirected to study this material. Because of the characterization capabilities available, we were able to establish quickly that the very large magnetoresistance depended exponentially on the magnetization (strong charge-spin coupling) and that coupling of the charge and spin to the lattice (polaron formation) was crucial for describing observations. These conclusions subsequently were verified by extensive additional measurements by us and others and were seminal discoveries leading 
to the now-accepted viewpoint for understanding the large magnetoresistance in these materials.

Accomplishments outlined above have substantially broadened our understanding of advanced materials in which electronic correlations, in particular the coupling among magnetic, electronic and lattice interactions, dominate physical properties. This understanding has application to broad classes of nuclear and advanced materials and has set new research directions at Los Alamos and elsewhere. 


\section{Publications}

1. Severing, A., Perring, T., Thompson, J. D., Canfield, P.C., Fisk, Z., "Spin-Hole Doping in the Kondo Insulator $\mathrm{Ce}_{3} \mathrm{Bi}_{4} \mathrm{Pt}_{3}$ Studied by Neutron Scattering," Physica B, 199-200, 480 (1994).

2. Heffner, R. H, "Muon Spin Relaxation Studies of the Interplay Between Magnetism and Superconductivity in Heavy Fermion Systems,"Journal of Alloys and Compounds, 213214, 232 (1994).

3. Beyermann, W. P., Heffner, R. H., Smith, J. L., Hundley, M. F., Canfield, P.C., Thompson, J. D., "Effects of Boron Substitution on the Superconducting State of UBe ${ }_{13}$," Phys. Rev. B, 51, 404 (1995).

4. Ahrens, E. T., Hammel, P.C., Heffner, R. H., Reyes, A. P., Smith, J. L., Clark, W.

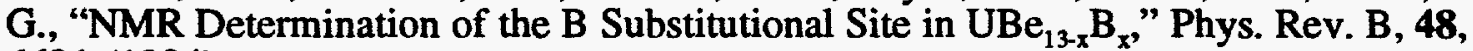
6691 (1994).

5. Reyes, A. P., Heffner, R. H., Canfield, P.C., Thompson, J. D., Fisk, Z., " ${ }^{209}$ Bi NMR and NQR Investigation of the Small-Gap Semiconductor $\mathrm{Ce}_{3} \mathrm{Bi}_{4} \mathrm{Pt}_{3}$," Phys. Rev. $\mathrm{B}, 49$, 16321 (1994).

6. Neiuwenhuys, G. J., Mentink, S. A. M., Menovsky, A. A., Amato, A., Feyerherm, R., Gygax, F. N., Heffner, R. H., Le, L. P., MacLaughlin, D. E., Schenck, A., " $\mu^{+} S R$ in Antiferromagnetic UNi $\mathrm{UN}_{4}$," Physica B, 206-207, 470 (1995).

7. Le, L. P., Heffner, R. H., Neiewenhuys, G. J., Canfield, P.C., Cho, B. K., Amato, A., Feyerherm, R., Gygax, F. N., MacLaughlin, D. E., Schenck, A., " $\mu$ 'SR Studies of Magnetic Properties of Boron Carbide Superconductors," Physica B, 206-207, 552 (1995).

8. Rainford, B. D., Adroja, D. T., Severing, A., Goremychkin, E. A., Thompson, J. D., Fisk, Z., "Inelastic Neutron Scattering Study of $\mathrm{U}_{3} \mathrm{Pt}_{3} \mathrm{Sb}_{4}$ " 206-207, 464 (1995).

9. Fisk, Z., Sarrao, J. L., Thompson, J. D., Mandrus, D., Hundley, M. F., Migliori, A., Bucher, B., Schlesinger, Z., Aeppli, G., Ott, H-R., Canfield, P.C., Brown, S., "Kondo Insulators," Physica B, 206-207, 798 (1995).

10. Heffner, R. H., Smith, J. L, Hundley, M. F., Canfield, P.C., Thompson, J. D., "Effects of Boron Substitution on the Superconducting State of $\mathrm{UBe}_{13}$," Physical Review B, 51, 404 (1995).

11. Ahrens, E. T., Heffner, R. H., Hammel, P.C., Reyes, A. P., Smith, J. L., Clark, W. G., ${ }^{" 9} \mathrm{Be}$ and ${ }^{11} \mathrm{~B}$ NMR Study of Superconductivity in Boron Doped $\mathrm{UBe}_{13}$," Physica B, 206-207, 589 (1995).

12. Reyes, A. P., Le, L. P., Heffner, R. H., Ahrens, E. T., Fisk, Z., Canfield, P.C.,

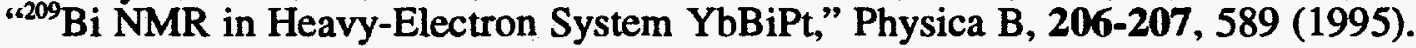

13. Feyerherm, R., Amato, A., Geibel, C., Gygax, F. N., Hellmann, P., Heffner, R. H., MacLaughlin, D. E., Muller-Reisener, R., Nieuwenhuys, G. J., Schenck, A., Steglich, $\mathrm{F}$., "On the competition between Superconductivity and Magnetism in $\mathrm{CeCu}_{2} \mathrm{Si}_{2}$," Physica B, 206-207, 596 (1995). 
14. Mandrus, D., Sarrao, J. L., Migliori, A., Thompson, J. D., Fisk, Z., "Thermodynamics of FeSi," Physical Review B, 51, 4763 (1995).

15. Cornelius, A., Schilling, J. S., Mandrus, D., Thompson, J. D., “Anomalous Hydrostatic Pressure Dependence of the Curie Temperature of the Kondo-Lattice Compound YbNiSn to $38 \mathrm{GPa}$," Physical Review B (Rapid Communication), 52, R15699 (1995).

16. Hundley, M. F.; Hawley, M., Heffner, R. H., Jia, Q. X., Neumeier, J. J., Tesmer, J., Thompson, J. D., Wu, X. D., "Transport-Magnetism Correlations in the Ferromagnetic Oxide $\mathrm{La}_{0.7} \mathrm{Ca}_{0.3} \mathrm{MnO}_{3}$," Applied Physics Letters, 67, 860 (1995).

17. Heffner, R. H., Norman, M. R., "Heavy Fermion Superconductivity," Comments on Condensed Matter Physics, 17, 361 (1996).

18. Hundley, M. F., Neumeier, J. J., Thompson, J. D., Lacerda, A., Canfield, P.C., "Magnetostriction and Thermal Expansion of the Kondo Semiconductor $\mathrm{Ce}_{3} \mathrm{Bi}_{4} \mathrm{Pt}_{3}$," Physical Phenomena at High Magnetic Fields-II, 2, 203 (1996).

19. Movshovich, R., Graf, T., Mandrus, D., Hundley, M. F., Thompson, J. D., Fisher, R. A., "Response of $\mathrm{CeRh}_{2} \mathrm{Si}_{2}$ to Pressure," Physica B, 223-224, 126 (1996).

20. Movshovich, R., Hundley, M. F., Neumeier, J. J., Thompson, J. D., Lawrence, J. M., Lacerda, A., Fisk, Z., "Magnetism in CePtPb," Physical Review B, 53, 5465 (1996).

21. Movshovich, R., Graf, T., Mandrus, D., Thompson, J. D., Smith, J. L., Fisk, Z., "Superconductivity in Heavy Fermion $\mathrm{CeRh}_{2} \mathrm{Si}_{2}$,"Physical Review B, 53, 8241 (1996).

22. Kwei, G. H., Lawrence, J. M., Sarrao, J. L., Fisk, Z., Mandrus, D., Thompson, J. D., "Structure and Disorder in YbInCu ${ }_{4}$ " Physical Review B, 54, 6011 (1996).

23. Sarrao, J. L., Immer, C.D., Benton, C. L, Fisk, Z., Lawrence, J. M., Mandrus, D., Thompson, J. D., "YbIn ${ }_{1-x} \mathrm{Ag}_{\mathrm{x}} \mathrm{Cu}_{4}$ : Evolution from First-Order Valence Transition to Heavy Fermion Behavior," Physical Review B, in press.

24. Mentink, S. A. M., Amitsuka, H., deVisser, A., Slanic, Z., Belanger, J. J., Neumeier, J. J., Thompson, J. D., Mydosh, J. A., Mason, J. E., "Thermodynamic Study of the Magnetic Phase Transition in $\mathrm{UNi}_{4} \mathrm{~B}$," Physica $\mathrm{B}$, submitted.

25. Heffner, R. H., Le, L. P., Nieuwenhuys, G. J., MacLaughlin, D. E., Amato, A., Gygax, F. N., Schenck, A., Stewart, G., Ott, H.R., "Possible Evidence for Spin or Orbital Magnetism below the Superconducting Transition Temperature in UBe ${ }_{12.91} B_{0.09}$," Physica B, submitted. 